\title{
Spatial Resolution of a Laboratory Based X-Ray Cone-Beam Laminography Scanning System for Various Trajectories
}

Hans Deyhle ${ }^{1}$, Hossein Towsyfyan ${ }^{1}$, Ander Biguri ${ }^{1}$, Mark Mavrogordato ${ }^{2}$, Richard Boardman ${ }^{2}$, Thomas Blumensath ${ }^{1,2 *}$

1. Institute of Sound and Vibration Research (ISVR), Signal Processing and Control Group, University of Southampton, Southampton, SO17 1BJ,UK

2. $\mu$-VIS X-Ray Imaging Centre, University of Southampton, Southampton, SO17 1BJ,UK

\begin{abstract}
Computed laminography (CL), a long established NDT method, is ideal for the non-destructive evaluation of plate-like structures, for which conventional computed tomography (CT) is less well suited. This paper demonstrates the feasibility of implementing raster CL on a Nikon Metrology custom build X-ray CT scanner without the need for specialised equipment. To provide a more principled way to demonstrate the widespread adoption of CL for defect characterization in plate-like structures, a test specimen was designed and manufactured from Polyoxymethylene (Delrin) that allows well defined features of different size and orientation to be characterized. The study highlights the relation between direction-dependent spatial resolution, feature geometry and scan trajectory. It demonstrates that high-resolution and highspeed in situ volume-imaging of plate-like structures with large aspect ratios is viable at levels that would be challenging via $\mathrm{CT}$ or alternative three-dimensional NDT methods.
\end{abstract}

Key words: Computed laminography; Resolution.

\section{Introduction}

X-ray computed tomography (CT) is one of the well-established and widely accepted imaging modalities in many different applications such as medical sciences [1, 2], animal imaging [3, 4], material science [5], geoscience [6,7] as well as many other engineering applications [8-10]. $\mathrm{CT}$ is also the gold standard in post-failure analysis for engineered components e.g. air crash investigations [11].

CT provides three-dimensional information with nominally isotropic resolution, from a macroscopic level $[12,13]$ down to a microscopic level (e.g. to the fibre architecture and porosity distribution in composite materials [14-16]) with a high accuracy. Tomographic information is acquired by taking a finite number of radiographic projection images systematically from equally spaced projection angles while an object is rotated more than $180^{\circ}$ with respect to an X-ray source and detector. Volumetric images are estimated by feeding the projections into a 'reconstruction

\footnotetext{
* Corresponding Author:

E-mail: Thomas.Blumensath@soton.ac.uk (T. Blumensath)
} 
algorithm', such as the filtered back projection (FBP) algorithm. FBP techniques provide fast reconstruction time [17], and works well if enough, low noise projection images are available. With more challenging data sets, alternative iterative reconstruction techniques can have significant advantages [18-20].

To acquire high resolution scans, CT is limited by the size and geometry of the objects. Traditional CT scanning is best suited to approximately cylindrical objects, but the technique is less well suited to the inspection of plate-like components/structures especially for high resolution imaging with cone-beam systems. There is now an increasing demand for three dimensional inspection of such components/structures including ball grid arrays [21], paintings [22, 23], printing circuit boards [24-26], fossils [27], and composite panels in the aerospace industry $[18,28,29]$. For instance, many aerospace composite panels are potentially very large but extremely flat structures, such as aircraft wings and tails and therefore have extremely high aspect ratios which are extended beyond the field of view of the detector. In such scans, if the path lengths through the scanned object vary dramatically at different angles, or if extreme differences in the object's diameters in longitudinal and transversal direction exist, scanning and reconstruction is challenging. Moreover, these structures may prevent a full rotation due to the risk of collision with the X-ray tube. Therefore, the structure has to be positioned away from the source resulting in decreased magnification and consequently a poorer resolution.

Several solutions to the problem of scanning plate-like objects with high aspect ratios have been explored, including dual-energy scanning [30-33], and region of interest (ROI) scanning [16, 17]. The former requires additional scanning time, and both solutions still require a full rotation of the object, and are thus limited to smaller components or lower resolution scans. An alternative is computed laminography (CL) which has recently received increasing attention [28, 29, 34-38]. In CL, the angular information is obtained by alternative trajectories which do not necessarily require full object rotation, in contrast to conventional $\mathrm{CT}$, where a full rotation is necessary. Research has been reported in the literature demonstrating the efficiency of CL to volumetric inspection of plate-like structures, mostly composite panels with high aspect ratios [39-41]. CL is thus a viable alternative to ultrasonic inspection, when inspecting hybrid materials, such as aluminum bonded to composites which are increasingly used in aerospace applications [42], and which are challenging to inspect with ultrasound due to multiple interfaces, variable and complex geometries and different elastic properties.

In laminographic scanning, relative motion between the source and the test object generates a range of scan trajectories [26, 28, 38, 40, 43-46]. Many of these scan trajectories allow laterally extended samples to be imaged at high-resolutions. Moreover, the technique provides similar X- 
ray transmission path lengths for each projection [18, 39, 47], increasing achievable image contrast.

The implementation of certain trajectories requires the relative movement of source and detector $[28,46]$. As calibrated motion of X-ray source and/or detector are often challenging, limited angle and rotary laminography are gaining more attention in industrial applications as they can be implemented on standard CT scanners by moving the sample only, Fig. 1.

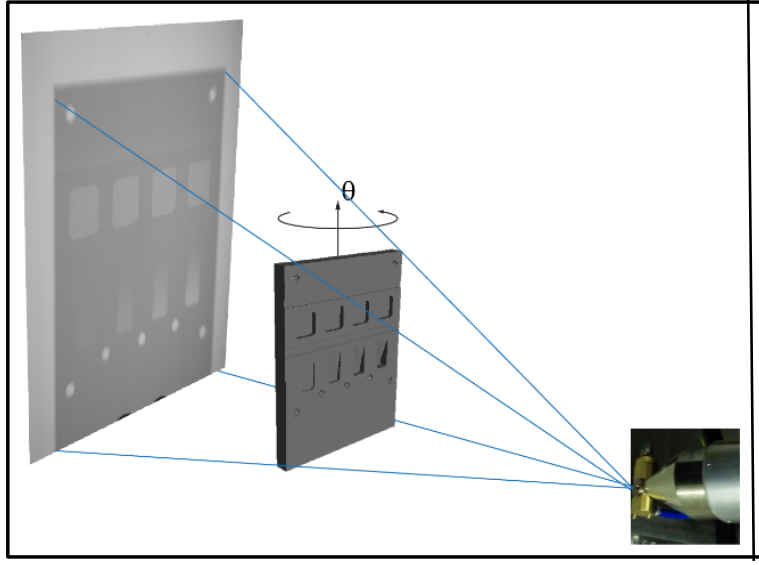

(a)

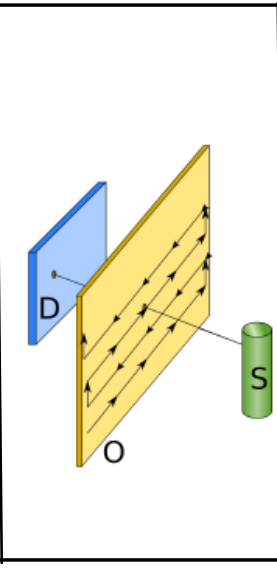

(b)

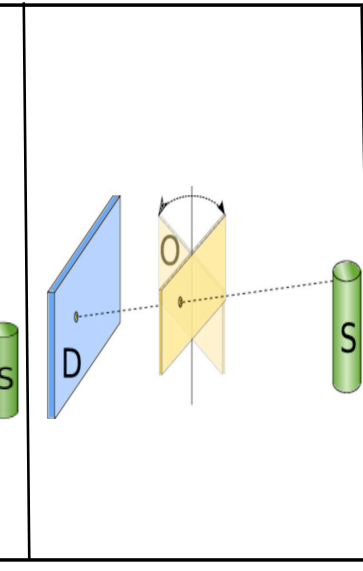

(c)

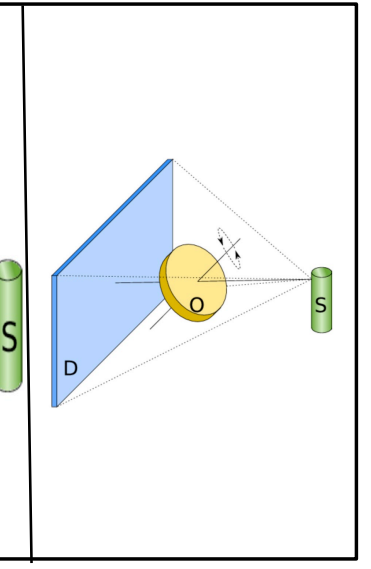

(d)

Fig. 1. Schematic diagram showing (a) Laminography set up (b) Raster trajectory [28], (c) Limited angle trajectory [28], (d) Rotary trajectory [28]. Note that $D, O$ and $S$ represent detector, object and source respectively.

In raster laminography, the sample is moved to positions on a grid of points in the plane orthogonal to the central X-ray beam and hence is well suited to scan relatively large components. Refs. $[29,46]$ report that raster laminography is able to detect sharp features that lie in the main plane of the motion, but features in the direction perpendicular to this are less well resolved. In limited angle laminography, the object is rotated through a smaller angular range, e.g. \pm 25 degrees, around an axis parallel to the plane of detector, whilst in the rotary laminography the test object is fully rotated but around an axis tilted relative to the plane of the detector. It has been reported in [45] that rotary laminography can produce reconstructions of superior quality in the plane parallel to the object face, when compared to limited-angle CT. The advantage of raster scan trajectories is that they allow inspection of large flat-like components, whilst limited angle and rotation based methods focus on a region of interest at a time. On the other hand, the use of a single rotation axis makes system calibration and post-scan geometric error correction easier.

However, the drawback of all CL methods is that, in contrast to CT, specific trajectory dependent directions within the object are not investigated, resulting in poor feature detectability/loss of information in said directions. It is therefore crucial to tune the trajectory of a CL system to 
balance the trade-off between the amount of acquired laminographic information against the weight dimension and geometry of the test object. Additionally, iterative reconstruction algorithms e.g. simultaneous iterative reconstruction technique (SIRT) or conjugate gradient least squares (CGLS) [48] generally provide better image quality [49, 50] compared to standard filtered back projection (FBP) methods for reconstruction [26], e.g. Feldkamp-Davis-Kress (FDK). Iterative reconstruction allows the use of additional prior information (e.g. CAD data) to guide the reconstruction $[48,51]$, however, at the expense of additional computation cost.

Initial experiments demonstrate benefits and drawbacks of different design options and scanning trajectories [29, 38, 44-46]. This paper uses a test specimen manufactured with features with different orientations and scales in order to provide a quantitative evaluation of anisotropy in reconstructed images, focusing particularly on raster and limited angle CL inspection of aerospace flat-like structures. The influence of different reconstruction methods is highlighted.

\section{Materials and Methods}

\subsection{Custom build X-ray CT scanner}

The imaging experiments have been carried out at the $\mu$-VIS X-ray Imaging Centre at the University of Southampton. A Nikon Metrology custom X-ray CT scanner that contains a 225kV micro focus X-ray source with a tungsten reflection target, a sample stage that moves in $x$ and $y$ direction and a $400 \mathrm{~mm}$ by $400 \mathrm{~mm}(2000 \times 2000$ pixel with $0.2 \mathrm{~mm}$ pixel pitch) high sensitivity flat panel detector (Perkin Elmer XRD 1621 CN3 EHS) was used to perform the experiments, Fig. 2. Motion in the $z$-direction was achieved by translating source and detector. Only the existing scanner motions (such as source, detector and sample stage motions) were used to generate the desired scanning trajectories.

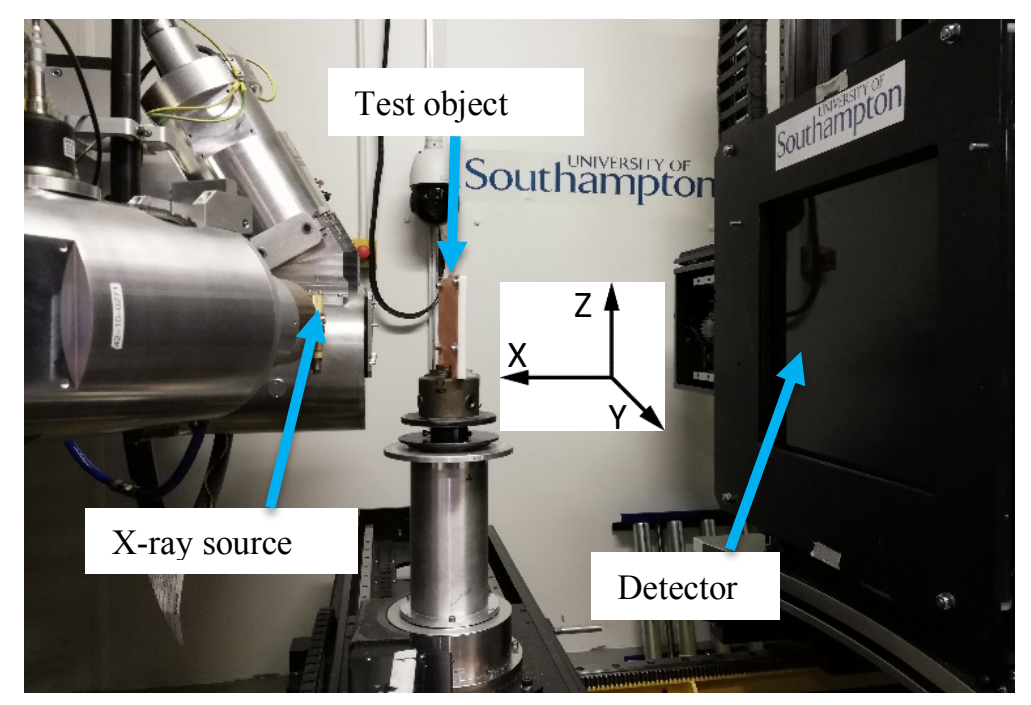

Fig. 2. Custom build walk-in bay X-ray CT scanner for laminography of the test specimen. 


\subsection{Sample design and manufacture}

To simulate aerospace composites, as one of the attractive applications of CL inspection, the test specimen was designed and manufactured from Polyoxymethylene (Delrin) which does not introduce significant scatter, and has a low X-ray attenuation coefficient. This specimen is a flat panel with high aspect ratio and has several features such as steps, wedges of different depth and measures $130 \mathrm{~mm} \times 100 \mathrm{~mm} \times 10 \mathrm{~mm}$ as detailed in Appendix A. Three specific features, as shown in Fig. 3, are characterised in the remainder of this paper. Three dimensional characterization of the manufactured test specimen is extremely difficult as it has several features in the thickness direction which are potentially less well resolved in subsequent reconstructions. Several inserts of different thicknesses i.e. an aluminum foam and touching pairs of hardened chrome steel ball bearings of different diameter $(0.5,1$ and $1.5 \mathrm{~mm})$, Fig. 4 , exist to examine the quality of CL reconstruction. Moreover, a copper meshes of A5 size (mesh number 30, $0.28 \mathrm{~mm}$ wire thickness) was provided to be mounted on one side of the specimen to simulate lightning strike protection as used on some aerospace composites. It was expected that the copper mesh, would introduce artefacts that would make the interpretation of the results more challenging.

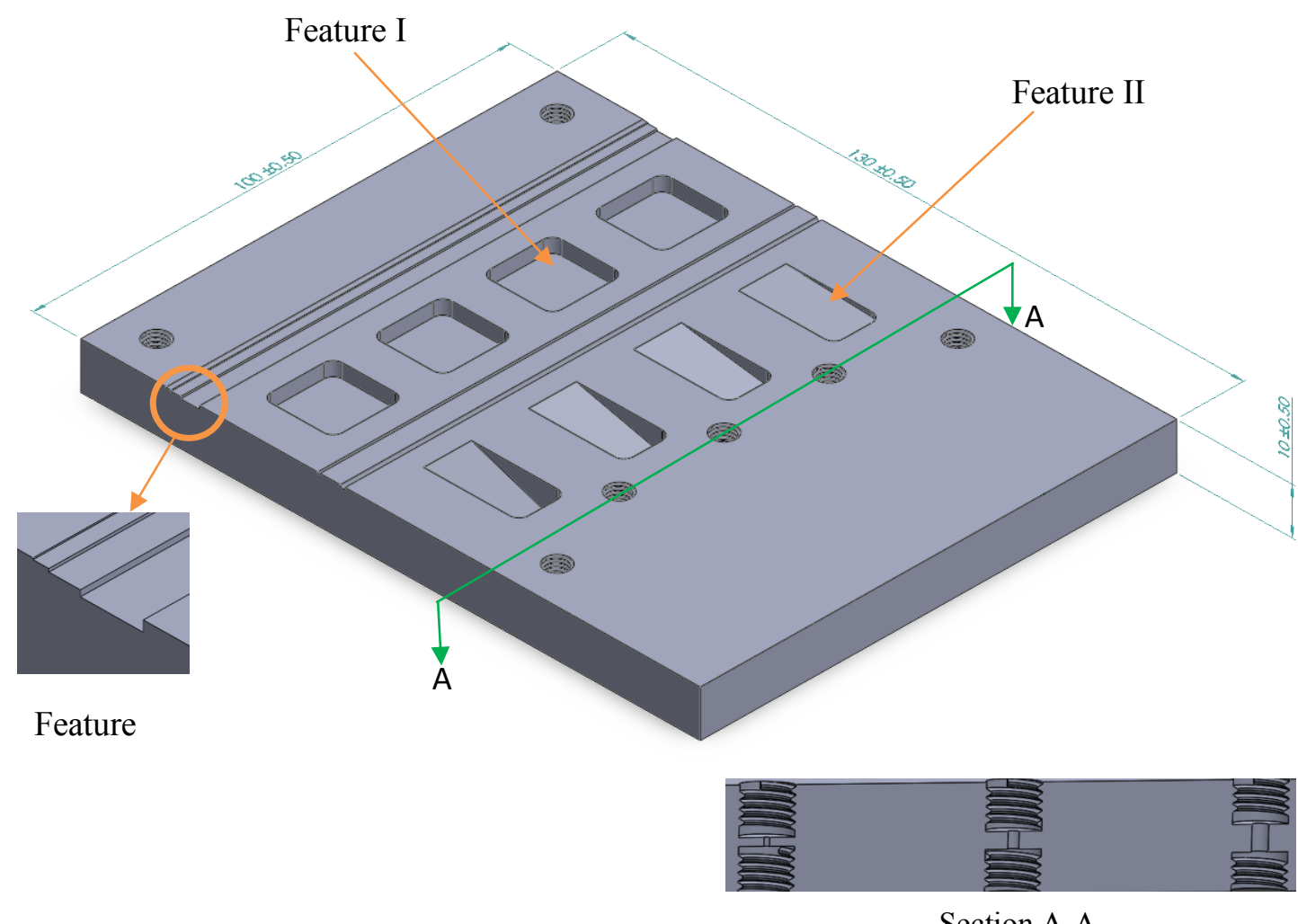

Section A-A

Fig. 3. The desired features of the test specimen. Cross-section A-A shows the arrangement for inserting and fixing the pairs of steel ball bearings, which are held in place using plastic grub screws inserted from both sides. 


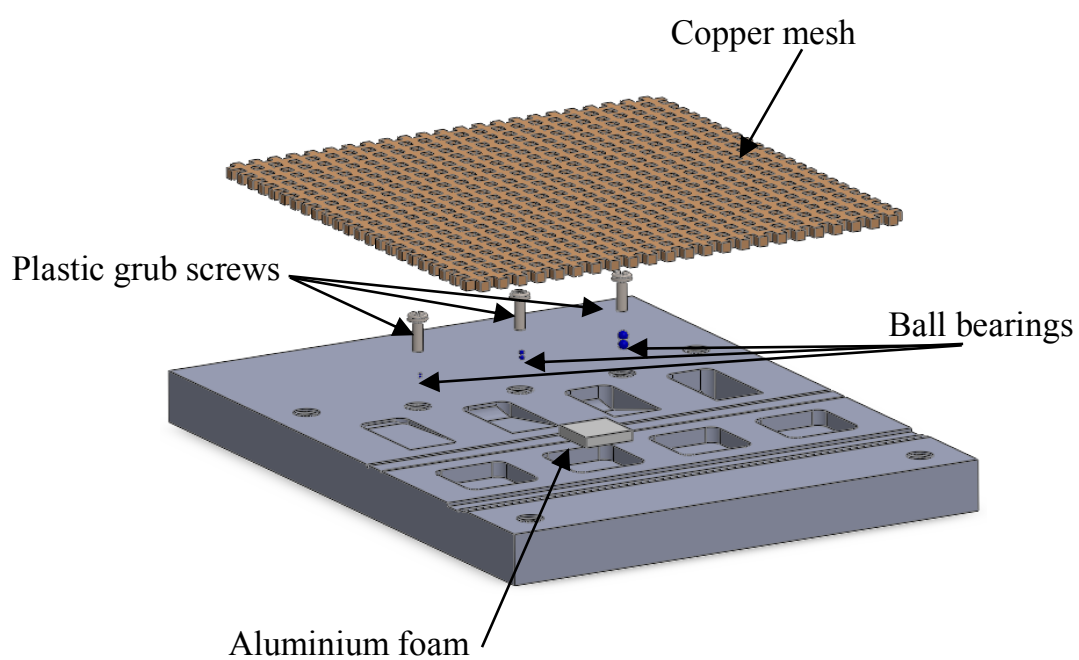

(a)

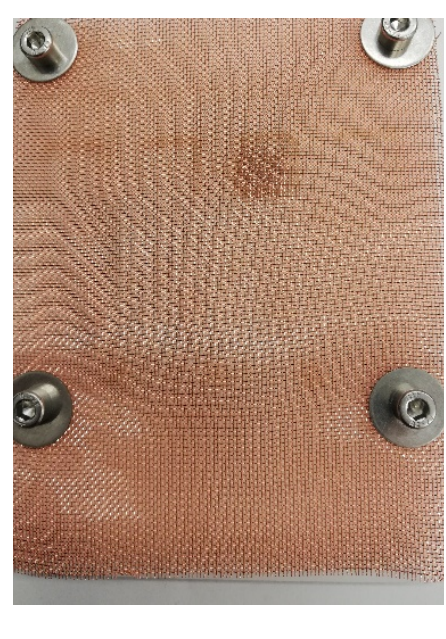

(b)

Fig. 4. The 3D layout of the test specimen after assembly: (a) Schematic illustration of inserts along with the copper mesh (b) The specimen after assembly.

\subsection{Laminographic data acquisition}

The sample was mounted on the rotation stage parallel to the detector and data was acquired using different laminographic trajectories as detailed in Table. 1. Each trajectory moved the sample linearly along a grid of points in the plane parallel to the detector. This motion was achieved using the scanner's linear axis which move the rotation stage (y-direction) and the source/detector assembly (z-direction). For trajectory A we repeated the same raster motion three times, but in each of these raster scans, we rotated the sample around the $\mathrm{z}$ axis to a different rotational offset. For all trajectories, the overall number of projections was 48 , apart for trajectory C where it was 49 .

In trajectory $\mathrm{A}$, the sample was moved in a line along the $\mathrm{y}$-axis and projections were taken at 16 equidistant points with a step size of $14.25 \mathrm{~mm}$, see Fig. 1(b). This scan was repeated for three angular positions around the rotation axis, using 0 degrees (parallel to the detector) and \pm 30 degrees, see Fig. 1(c). This trajectory samples the largest angular range among the proposed trajectories and can be considered as a combination of raster and limited angle trajectories.

Trajectory B also moved the sample in a line, this time using a step size of $4.75 \mathrm{~mm}$, but without the rotational steps. In Trajectory $\mathrm{C}$, the test object was moved to positions on a regular $7 \times 7(y$ and $z$ directions) grid of points in the plane orthogonal to the X-ray beam with grid spacing of $33.25 \mathrm{~mm}$. Trajectory D was similar to trajectory B, but with movement in the $z$-direction. 
In all of the above experiments, the source voltage was set to $120 \mathrm{kV}$ with power adjusted to $23 \mathrm{~W}$ and the exposure time per projection was $134 \mathrm{~ms}$. The source to detector distance was set to $802 \mathrm{~mm}$ whereas the source to specimen distance was $255 \mathrm{~mm}$, resulting in a geometrical magnification of 3.14, an effective pixel size of $63.7 \mu \mathrm{m}$, and field of view of $127.4 \mathrm{~mm}$. The cone angle in the $\mathrm{x}-\mathrm{y}$ plane covered by the detector was $\pm 12.25^{\circ}$.

\begin{tabular}{ccccc}
\hline Trajectory & $\begin{array}{c}\text { Number of } \\
\text { points in Y } \\
\text { direction }\end{array}$ & $\begin{array}{c}\text { Number of } \\
\text { points in Z } \\
\text { direction }\end{array}$ & $\begin{array}{c}\text { Rotation } \\
\text { angle } \\
\text { (degrees) }\end{array}$ & $\begin{array}{c}\text { Number of } \\
\text { projections }\end{array}$ \\
\hline A & 16 & 1 & $0, \pm 30$ & 48 \\
\hline B & 48 & 1 & 0 & 48 \\
\hline C & 7 & 7 & 0 & 49 \\
D & 1 & 48 & 0 & 48
\end{tabular}

Table. 1. The scanned trajectories applied in the experiments

Additionally, a region of interest (ROI) of the specimen containing the ball bearings was scanned with higher resolution. Here, the effective pixel size was $23 \mu \mathrm{m}$. The trajectory consisted of a $800 \times 3(y-z)$ grid of points. Other scan parameters were kept as in the previous scans. A third scan was performed with the copper mesh attached to the specimen using trajectory A (the pixel size was $0.0637 \mathrm{~mm}$ ). To study the effect of an increased number of projections and finer sampling, the specimen was scanned in a $24 \times 24$ regular raster grid with step size of $9.5 \mathrm{~mm}$ in $y$ and $z$ at three angular positions (i.e. $0^{0}$ and $\pm 30^{\circ}$ ).

Nikon Metrology`s Inspect-X version 2 was used to perform scan trajectories. Custom macros were written in Visual Basic for Applications (VBA) that generated the coordinates of each point in the trajectory and request the system manipulator move to that point. After each move, a radiograph was acquired and metadata written (including the requested and reported position of the system manipulators, as well as X-ray beam and imaging system parameters).

\subsection{Reconstruction}

Images were reconstructed using Feldkamp-Davis-Kress (FDK) [52] and CGLS [48, 51] algorithms implemented in the TIGRE toolbox [53, 54]. In FDK, generally a 1D high-pass filter in the direction perpendicular to the axis of rotation used. In this work, the data were filtered with a 2D Fourier ramp filter instead to account for the possible virtual rotation around the horizontal axis in trajectories C and D. For consistency and comparability, all datasets were filtered with the same kernel. Prior to filtering, the projections were padded to 2048 pixels by repeating the 
edge values. This reduced line artefacts common in raster laminographic scans [29]. CGLS was used due to its fast convergence. Vertical line artefacts in CGLS were removed after reconstruction. To this end, we chose a horizontal slice containing no features and subtracted it from all slices in the dataset.

\subsection{Geometric calibration}

Laminographic data reconstruction relies on the precise knowledge of the system and scan geometry. In particular, we need to know the relative positions and orientations of source, object and detector. For our scan trajectories, we thus need to know the exact orientation of the different linear axis in the system as well as the position of the rotation axis relative to these. Whilst these parameters can be estimated from a knowledge of the system geometry and the encoder values provided by the system, we conducted a separate calibration scan to provide better estimates of these geometric parameters.

One approach for geometric calibration of tomosynthesis systems uses a calibrated phantom with markers at known positions [55-57]. This method, however, relies on the availability of a calibrated phantom with markers at known locations. Instead, an approach based on Ref. [58] is used here where one or several markers with unknown relative positions are imaged but with the manipulator in different configurations. These projection images contain sufficient information for an accurate characterization of the system geometry, including test object location, source location, detector location and orientation and the alignment of linear and rotational manipulator axes. The accuracy of this method relies on the manipulator system's accuracy which was found in [58] to allow the geometry to be estimated with sufficient accuracy to guarantee average geometric errors in the projected image to be below pixel size.

To perform geometric calibration, three small high precision chrome steel spheres $(6 \mathrm{~mm}$ diameter, grade 10 hardened) were glued onto an X-ray transparent plastic tube to form the calibration phantom. The tube then was mounted on the system manipulator. To calibrate the linear axis, nine projections at two different magnifications were acquired in a raster configuration as shown in Fig.5 (a) and (b). For rotational axis calibration, the manipulator was rotated through a range of rotational offsets $\left(60^{\circ}\right)$, Fig.5 (c). Geometric information was then extracted as described in [58]. 

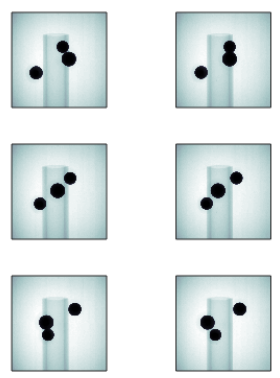

(a)
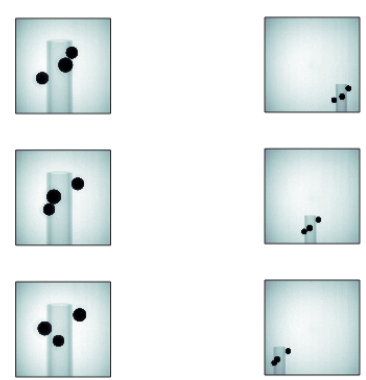
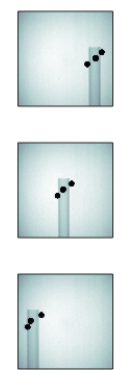

(b)
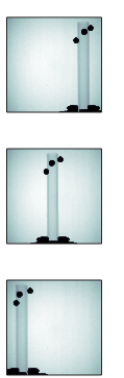

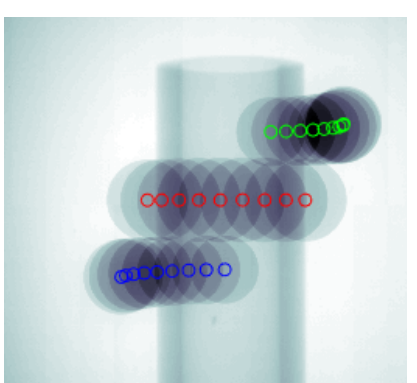

(c)

Fig. 5. The calibration scans (a) Magnification 3.14 (b) Magnification 2 (c) Rotation at magnification of 3.14 .

\section{Result and discussion}

\subsection{Edge response as function of trajectory and reconstruction algorithm}

\subsubsection{Feature I}

A vertical slice through the dataset reconstructed with the FDK algorithm (left) and 10 iterations of CGLS (right), using trajectory B, is shown in Fig. 6 (a). The analyzed region of the slice is highlighted by the yellow square. We found that CGLS converged with a good accuracy after 10 iterations. Running more iterations (i.e. 20 and 40) results in no significant improvement in the image quality. Three orthogonal cuts through the region of interest are shown in Fig. 6 (b). Line profiles along the horizontal line (red, dashed) and vertical line (blue, dot-dashed) are shown in the graph. As it is evident, no clear edge can be identified in $x$-direction due to the poor spatial resolution along the beam direction. In order to compare the effectiveness of the studied trajectories, the spatial resolution is calculated from the $10 \%$ modulation transfer function (MTF) value of straight edges in $x, y$ and $z$ directions, as shown in Table. 2 for both FDK and CGLS.

In Fig. 6 (d) the line spread function (LSF) was calculated as derivative from the line profile across the edge as shown in Fig. 6 (c). The MTF, shown in Fig. 6 (e) was obtained from the power spectrum of the LSF. The spatial frequency where the MTF drops to $10 \%$ of the zero component was chosen as the measure of spatial resolution. Despite the lack of a clear edge in the $x$-direction, the method is still valid as long as the spatial resolution is better than a threshold.

Higher sampling in a specific direction leads to improved resolution in the same direction whereas a larger angular range improves the resolution in beam direction. For trajectories A and $\mathrm{B}$, no scanning was performed in the $z$ direction, resulting in the slightly poorer resolution in this direction compared to $y$, roughly $20 \%$ for A and $45 \%$ for B. Conversely, the resolution in $z$ 
improves in trajectories $\mathrm{C}$ and $\mathrm{D}$, at the cost of resolution in $y$. This demonstrates that the resolution highly depends on the trajectory chosen.
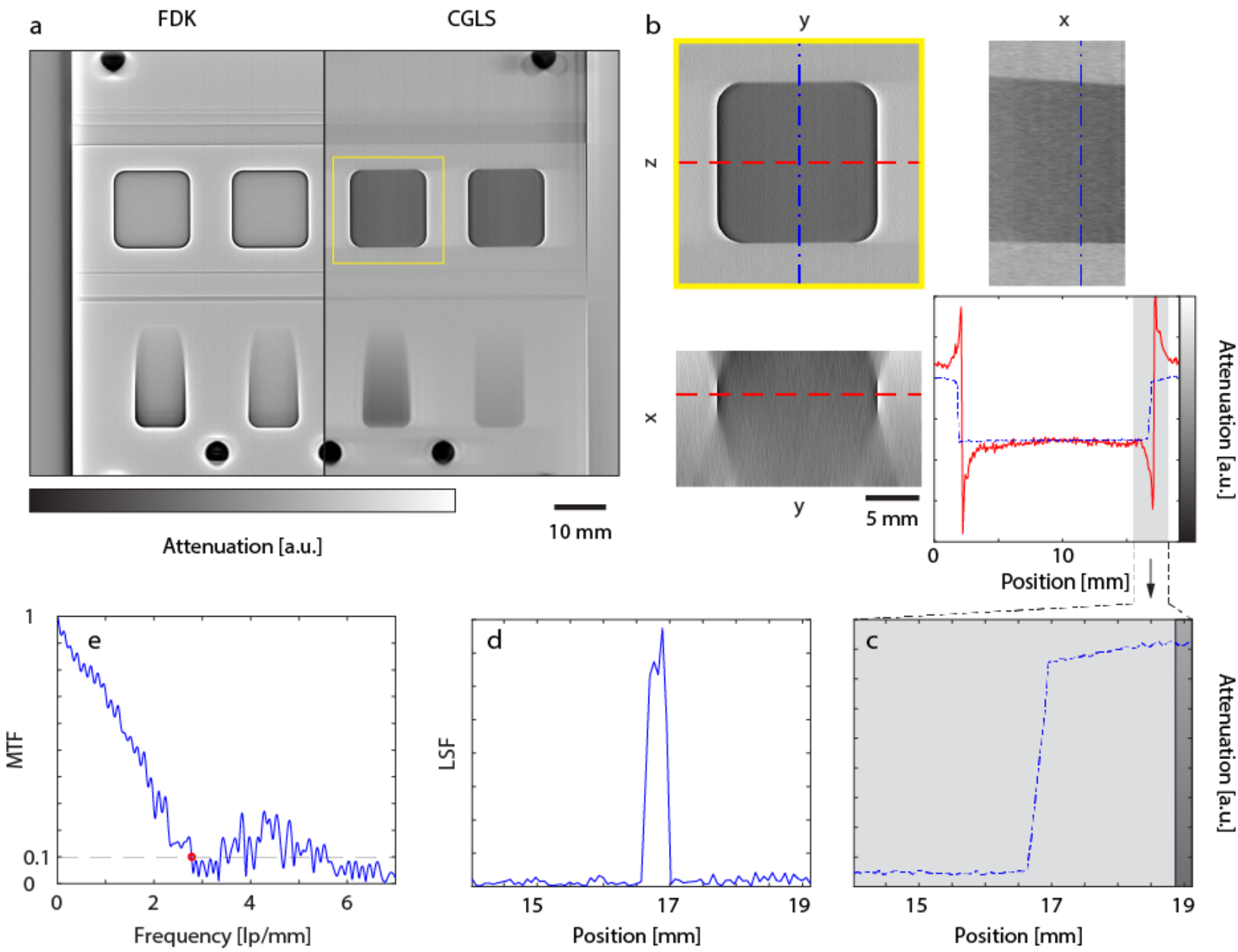

Fig. 6. The calculation of square window, (a) Vertical slice through the dataset reconstructed with the FDK (left half) and CGLS (right half), (b) Three orthogonal cuts through the analyzed region, (c) Line profile across an edge, (d) The line spread function, (e) The modulation transfer function.

Resolution in the beam direction, i.e. $x$-direction, is linked to the angular range covered. In trajectories B-D, the angular range is determined by the cone angle of $24.5^{0}$, whereas trajectory A, where the specimen was scanned at three different angles, covered an angular range of $73.5^{\circ}$ resulting in improved resolution in $x$ direction. This supports the choice of trajectory depending on the required resolution in any specific direction. The $2 \mathrm{D}$ filter kernel used for FDK reconstruction leads to edges along the $\mathrm{y}$ and $\mathrm{z}$ directions being enhanced independent of trajectory chosen. This can lead to the MTF approach failing, as was the case for the $y$-direction in trajectory D, see Table. 2. Additionally, the tabulated values obtained for the FDK in beam direction are unreasonably small, indicating the method is unreliable to determine spatial resolution in this case. Fig. 7 shows attenuation line profiles in beam direction across the dataset for both reconstructions. Whereas CGLS yields monotonically increasing attenuation values 
consistent with a highly out of focus edge, the FDK reconstruction results in a wavy curve not suitable for MTF analysis.

Table. 2. Spatial resolution in three orthogonal direction

\begin{tabular}{lllllll}
\hline Trajectory & \multicolumn{2}{l}{$\begin{array}{l}\text { Resolution in X } \\
\text { direction }(\mathrm{mm})\end{array}$} & \multicolumn{2}{l}{$\begin{array}{l}\text { Resolution in } \mathrm{Y} \\
\text { direction }(\mathrm{mm})\end{array}$} & \multicolumn{2}{l}{$\begin{array}{l}\text { Resolution in Z } \\
\text { direction }(\mathrm{mm})\end{array}$} \\
\cline { 2 - 7 } & CGLS & FDK & CGLS & FDK & CGLS & FDK \\
\hline $\mathrm{A}$ & 1.6316 & 0.3279 & 0.1026 & 0.0891 & 0.1214 & 0.0999 \\
\hline $\mathrm{B}$ & 7.2516 & 0.1925 & 0.0796 & 0.0686 & 0.1155 & 0.098 \\
\hline $\mathrm{C}$ & 6.5264 & N/A & 0.1514 & 0.0682 & 0.1225 & 0.0691 \\
\hline $\mathrm{D}$ & 6.5264 & 0.3490 & 0.0917 & N/A & 0.0771 & 0.0783
\end{tabular}

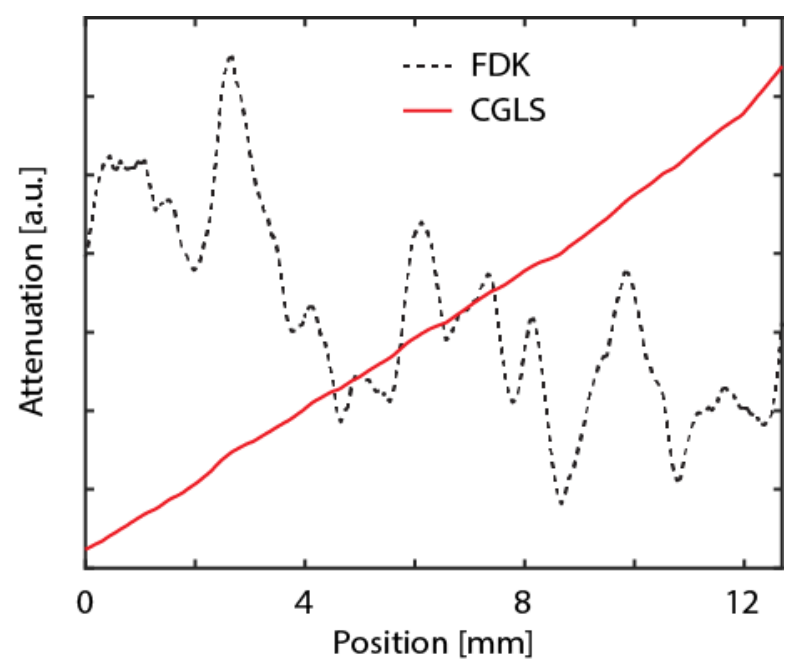

Fig. 7. Line profiles across the $x$-direction for trajectory B

\subsubsection{Feature II}

Another feature of interest is the wedge with the smallest depths, see Feature II in Fig. 3, which is characterized in Fig. 8 using a similar approach presented in previous section. For each line in the volume of interest (VOI), the 10\% MTF in y-direction as exemplified in Fig. 8 (a) is used as an indicative of how well the edges are reproduced.

A vertical slice through the VOI and a line profile along the dashed line are shown in Fig. 8 (b). The red line is a smoothed version of the black line (Gaussian kernel, sigma of 1.5 pixels). The in-plane gradient of the slice presented in Fig. 8 (b) is shown in Fig. 8 (c), which is equivalent to the LSF. The spatial resolution in line pairs per $\mathrm{mm}(\mathrm{lp} / \mathrm{mm})$ derived from the $10 \% \mathrm{MTF}$ value for each line in the dataset is shown for trajectories A through D in Figs. 8 (d-g) for the GCLS and Figs. 8 (h)-(k) for the FDK, indicating how well the location of features perpendicular to the beam direction can be estimated depending on their extension in the beam direction. The crosssection of the feature, obtained from the CAD data, is indicated in yellow. 
For CGLS, trajectory A best matches the ground truth, with MTF $10 \%$ values well above the background level for an edge depth above $\sim 0.5 \mathrm{~mm}$ inside the yellow area, and on background level outside. Thus, features with an extension above $0.5 \mathrm{~mm}$ in beam direction are faithfully reproduced, indicating that the spatial resolution in beam direction is even better than estimated in Table 2. Trajectory B overestimates the actual depth of the wedge (by a factor of approximately 3). For the other trajectories, the edge is not well reproduced, showing that sampling perpendicular to the edge was insufficient in these cases. In contrast to this, FDK does not work well for any of the trajectories. 

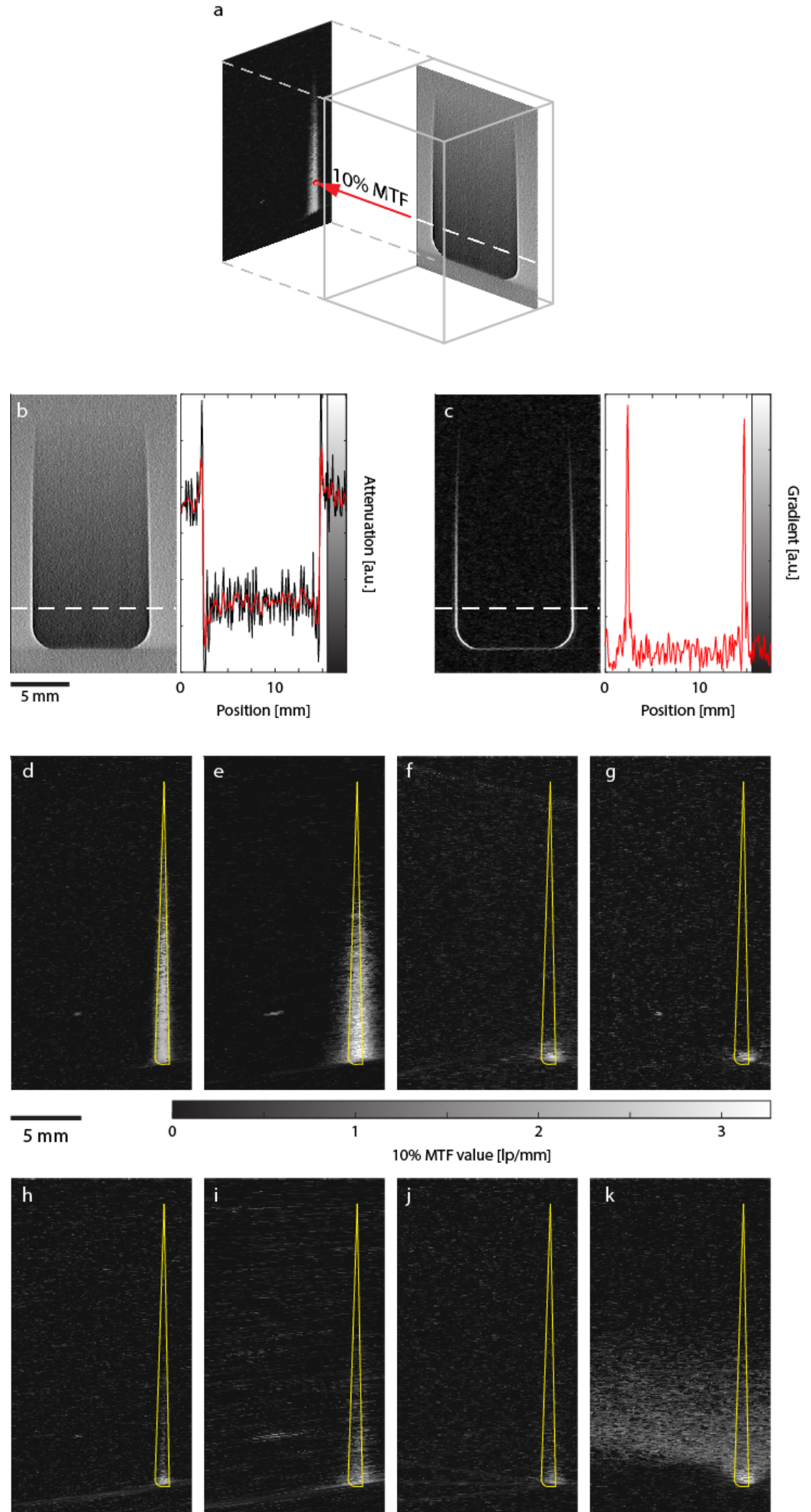

$10 \%$ MTF value $[\mathrm{lp} / \mathrm{mm}]$

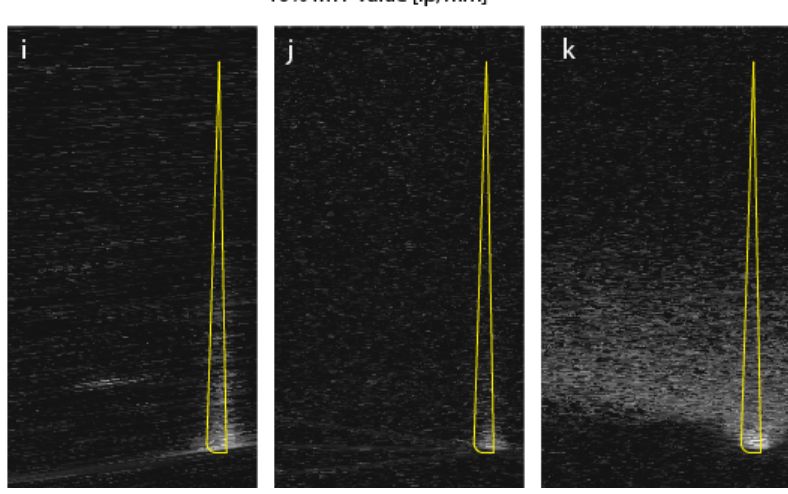

Fig. 8. Characterization of the wedges (a) The vertical slice through the volume of interest, (b) the attenuation line profile along the white dashed line (c) In-plane gradient of the line profile (d)-(g) The 10\% MTF value derived from the datasets reconstructed with 10 iterations of CGLS, trajectories A through D, (h)-(k) The 10\% MTF value derived from the datasets reconstructed with 10 iterations of FDK, trajectories A through D. 


\subsection{Feature detectability in beam direction}

\subsubsection{Feature III}

This section characterizes the resolution in beam direction by analyzing the laminographic data acquired for the step feature (Feature III in Fig. 3). As detailed in Appendix A, three steps with $1 \mathrm{~mm}, 2 \mathrm{~mm}$ and $4 \mathrm{~mm}$ depth are located in the beam direction. Fig. 9 presents the $x-z$ cross section through the steps along with related line profiles at varying depths. The brown (solid) line corresponds to a depth where all step walls are in focus, whereas the dashed lines are shifted $2.5 \mathrm{~mm}$ in beam direction in positive and negative direction, respectively. Note that in the graphs, they are artificially shifted in the vertical direction for clarity. For trajectories A and B, no clear delimitation of the step walls is visible due to the fact that the scanning direction was parallel to the step extension in $y$. Note that in trajectory A, the step wall starts to blur at a distance from the steps as indicated by the arrow in Fig. 9 (a) due to the higher angular range in this trajectory.

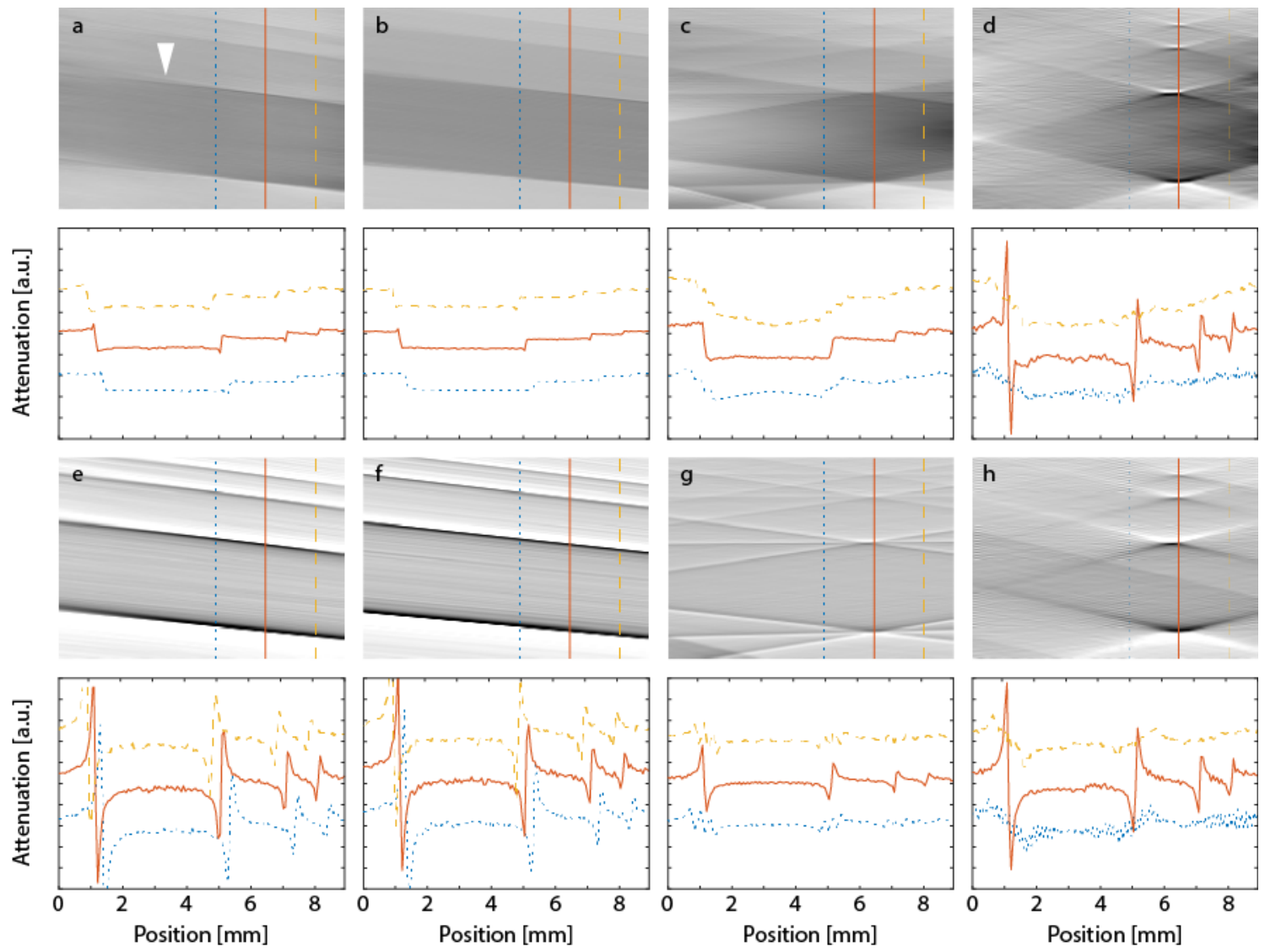

Fig. 9. Characterization of the steps (a)-(d) The datasets reconstructed with 10 iterations of CGLS, trajectories A through D, (e)-(h) The datasets reconstructed with FDK, trajectories A through D.

Trajectories $\mathrm{C}$ and $\mathrm{D}$ reproduce better response to the changes of the step heights, where trajectories were partially and entirely perpendicular to the edge extension, respectively. This is exemplified by the line profiles, where the step walls become less visible with increasing distance 
from the feature for trajectories $\mathrm{C}$ and $\mathrm{D}$. This insight is consistent with the results shown in Fig 8. Edges are best represented when the trajectory is perpendicular to the edge.

\subsubsection{Ball bearings}

As the balls are touching, the center to center distance is known. Determining the center to center distance in the reconstructed data is, therefore, an additional way of characterizing the resolving power in the beam direction. Figs. 10 (a) and (b) show vertical $(y-z)$ and horizontal $(x-y)$ slices through the CGLS reconstructed data of the ROI scan. Note the considerable amount of artefacts, due to the slice not passing through the ball bearings. In Figs. 10 (c)-(d), the attenuation line profiles along with the related standard deviation are shown, in order to determine the center-tocenter distance. The standard deviation can be considered a measure of sharpness of an image [59]. Thus, it will be highest where the ball bearings come into focus. As shown in Figs. 10 (c)(d), it is able to characterize the center to center distance for the largest and middle size bearings reasonably well, however, it fails to correctly characterize the center-to-canter-distance of the $0.5 \mathrm{~mm}$ bearings.
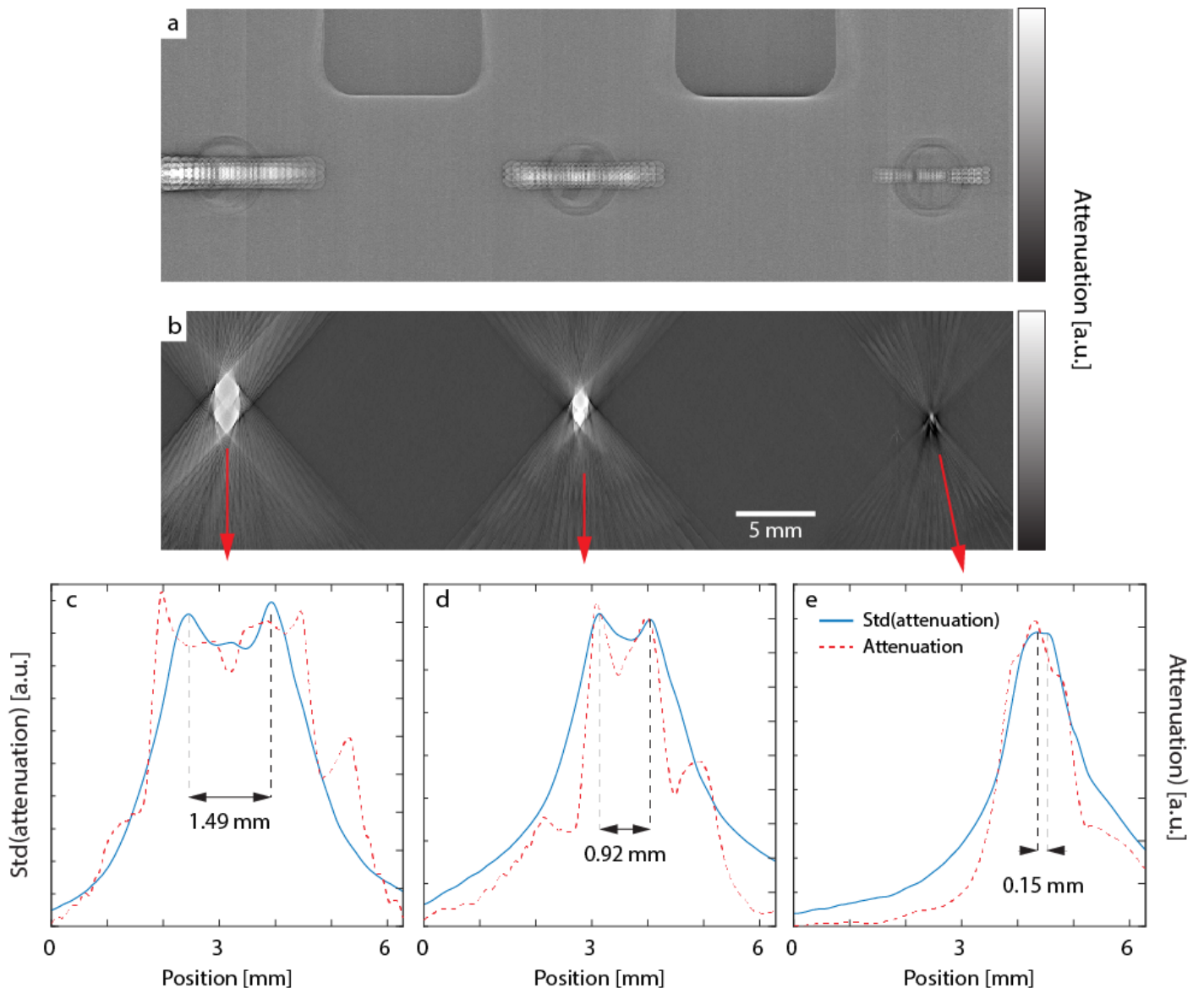

Fig. 10. Characterization of the ball bearings. (a) Top view slice (b) Front view slice (c) Attenuation line profile for the $0.5 \mathrm{~mm}$ diameter ball (d) Attenuation line profile for the $1.0 \mathrm{~mm}$ diameter ball (e) Attenuation line profile for the $1.5 \mathrm{~mm}$ diameter ball. 


\subsection{Reconstruction of more challenging datasets}

Composites panels in aerospace applications are often covered with a copper mesh lightning strike protection. To examine the effects of this additional component, a copper mesh was added on the specimen and an additional scan performed with the mesh in place as shown in Fig. 4. In $\mathrm{X}$-ray laminographic imaging, this produces visible artefacts throughout the reconstructed volume.

To compare the robustness of FDK and CGLS in reconstructing of more challenging data sets, the whole volume of the test object was reconstructed using trajectory A. At magnification of 2 . The scan parameters were kept the same as in Section 2.3. Fig. 11 shows slices that are roughly $5 \mathrm{~mm}$ away from the copper mesh, reconstructed with FDK (a) and in CGLS (b and c). Strong artefacts generated by the copper mesh are observed in the FDK reconstruction that do not allow any of the features to come in focus. CGLS reconstruction shows a marked improvement and also allows identifying the aluminum foam, indicated by a yellow square in the Figs. 11 (b) and (c). Increasing the number of projection by a factor 36, Fig. 11 (c), further reduces the severity of the artifacts. However, it comes at the expense of higher scanning time. Due to the artifact introduced by the copper mesh, resolution measurements similar to those discussed above, were not feasible here.
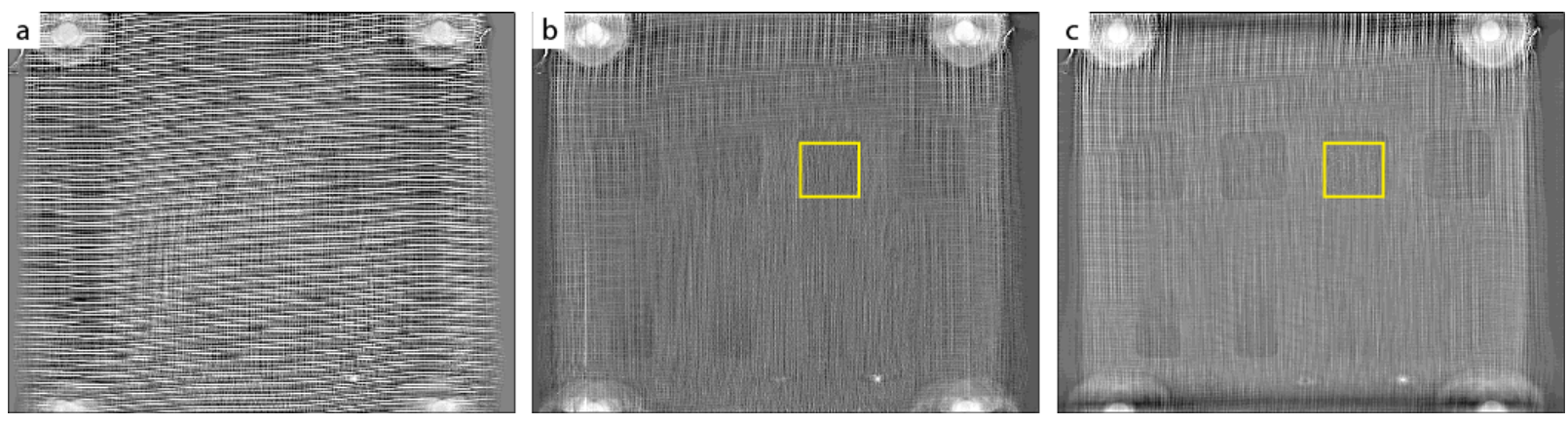

Fig. 11. Comparison of a same slice in FDK and CGLs. (a) Reconstructed by FDK 48 projections (b) Reconstructed by CGLS 10 iterations 48 projections (c) Reconstructed by CGLS 10 iterations 1728 projections.

\section{Conclusion}

A lab-based laminography system that allows the study of a wide range of laminographic scan trajectories was implemented. The exploratory experiment in the present work has shown that the implemented trajectories serve as a reasonable basis from which to proceed and perform relatively short scans, including ROI scans, and subsequent reconstruction for the characterization of features in the designed test specimen. We here focused on different methods 
to evaluate anisotropic spatial resolution. The analysis of results produces the following key points:

1. It was demonstrated that enough information can be acquired by computed laminography to characterise certain structural features in plate-like objects with high aspect ratio.

2. The conjugate gradient least squares (CGLS) reconstruction algorithm improved image quality compared to standard filtered back projection (FBP) methods, e.g. Feldkamp-Davis-Kress (FDK). The quality of the data reconstructed with FDK proved insufficient for the reliable characterization of features in the beam direction.

3. The edges of features were well reproduced, however resolution depends on relative orientation of edges and scan trajectory: trajectories perpendicular to edge features improved feature characterization.

4. Higher sampling in a specific direction lead to improved resolution in that same direction whereas a larger angular range improves the resolution in the beam direction. Therefore, a wider angular range coupled with a higher sampling rate is desirable.

5. Adding a copper mesh on the top surface of the specimen to simulate lightning strike protection was extremely detrimental to image quality. CGLS reconstruction, once again, shows a notable improvement.

\section{Acknowledgment}

The authors gratefully acknowledge the support of NVIDIA Corporation with the donation of the Titan Xp GPU used for this research. This research was supported by EPSRC grant EP/R002495/1 and the European Metrology Research Programme through grant 17IND08. 


\section{References}

1. Kalender, W.A., X-ray computed tomography. Physics in Medicine \& Biology, 2006. 51(13): p. R29.

2. Ritman, E.L., Current status of developments and applications of micro-CT. Annual review of biomedical engineering, 2011. 13: p. 531-552.

3. Paulus, M.J., et al., A review of high-resolution X-ray computed tomography and other imaging modalities for small animal research. Lab animal, 2001. 30(3): p. 36-45.

4. Chun, I.K., et al., X-ray micro-tomography system for small-animal imaging with zoom-in imaging capability. Physics in Medicine \& Biology, 2004. 49(17): p. 3889.

5. Maire, E., et al., On the application of X-ray microtomography in the field of materials science. Advanced Engineering Materials, 2001. 3(8): p. 539-546.

6. Ketcham, R.A. and W.D. Carlson, Acquisition, optimization and interpretation of X-ray computed tomographic imagery: applications to the geosciences. Computers \& Geosciences, 2001. 27(4): p. 381-400.

7. Cnudde, V. and M.N. Boone, High-resolution X-ray computed tomography in geosciences: A review of the current technology and applications. Earth-Science Reviews, 2013. 123: p. 1-17.

8. Garcea, S., Y. Wang, and P. Withers, X-ray computed tomography of polymer composites. Composites Science and Technology, 2017.

9. Stock, S.R., Recent advances in X-ray microtomography applied to materials. International Materials Reviews, 2008. 53(3): p. 129-181.

10. Amos, M., Advanced industrial x-ray computed tomography for defect detection and characterisation of composite structures. 2010, University of Manchester.

11. Griffioen, H., Air Crash Investigations: The Crash of American Airlines Flight 587.

12. Standards, B., BS ISO 16016-1 Non destructive testing. Radiation method. Computed tomogaphy Part 2. Principle, equipment and samples., in Technical report,British Standards, November 2009a.

13. Kastner, J., Special issue on the 6th conference on industrial computed tomography 2016 (iCT2016). Case studies in nondestructive testing and evaluation, 2016(6): p. 2-3.

14. Little, J.E., X. Yuan, and M.I. Jones, Characterisation of voids in fibre reinforced composite materials. NDT \& E International, 2012. 46: p. 122-127.

15. Reh, A., et al. Porosity maps-interactive exploration and visual analysis of porosity in carbon fiber reinforced polymers. in Computer Graphics Forum. 2012. Wiley Online Library.

16. Hernández, S., et al., Effect of curing cycle on void distribution and interlaminar shear strength in polymer-matrix composites. Composites science and technology, 2011. 71(10): p. 1331-1341.

17. Pan, X., E.Y. Sidky, and M. Vannier, Why do commercial CT scanners still employ traditional, filtered back-projection for image reconstruction? Inverse problems, 2009. 25(12): p. 123009.

18. Rouse, J.E., Characterisation of impact damage in carbon fibre reinforced plastics by $3 \mathrm{D}$ X-ray tomography. 2012.

19. Rezvani, N., Iterative reconstruction algorithms for polyenergetic x-ray computerized tomography. 2012, University of Toronto (Canada).

20. Jiang, M. and G. Wang, Convergence of the simultaneous algebraic reconstruction technique (SART). IEEE Transactions on Image Processing, 2003. 12(8): p. 957-961.

21. Moore, T.D., D. Vanderstraeten, and P.M. Forssell, Three-dimensional X-ray laminography as a tool for detection and characterization of BGA package defects. IEEE Transactions on Components and Packaging Technologies, 2002. 25(2): p. 224-229.

22. Legrand, S., et al., Examination of historical paintings by state-of-the-art hyperspectral imaging methods: from scanning infra-red spectroscopy to computed X-ray laminography. Heritage Science, 2014. 2(1): p. 13.

23. Janssens, K., et al., Non-invasive and non-destructive examination of artistic pigments, paints, and paintings by means of X-ray methods, in Analytical Chemistry for Cultural Heritage. 2017, Springer. p. 77-128.

24. Kim, H.K., et al. X-ray laminographic application of lens-coupled CMOS detector for PCB inspection. in 2001 IEEE Nuclear Science Symposium Conference Record (Cat. No. 01CH37310). 2001. IEEE. 
25. Cho, M.K., et al., Cone-beam digital tomosynthesis for thin slab objects. NDT \& E International, 2012. 47: p. 171-176.

26. Maisl, M., F. Porsch, and C. Schorr. Computed laminography for x-ray inspection of lightweight constructions. in 2nd International Symposium on NDT in Aerospace. 2010.

27. Houssaye, A., et al., Three-dimensional pelvis and limb anatomy of the Cenomanian hind-limbed snake Eupodophis descouensi (Squamata, Ophidia) revealed by synchrotron-radiation computed laminography. Journal of Vertebrate Paleontology, 2011. 31(1): p. 2-7.

28. O'brien, N., et al., Comparing cone beam laminographic system trajectories for composite NDT. Case studies in nondestructive testing and evaluation, 2016. 6: p. 56-61.

29. Wood, C.E., et al., Computed laminography of CFRP using an X-ray cone beam and robotic sample manipulator systems. IEEE Transactions on Nuclear Science, 2019.

30. Pfeiffer, F., et al., Tomographic reconstruction of three-dimensional objects from hard X-ray differential phase contrast projection images. Nuclear Instruments and Methods in Physics Research Section A: Accelerators, Spectrometers, Detectors and Associated Equipment, 2007. 580(2): p. 925-928.

31. Petersilka, M., et al., Technical principles of dual source CT. European journal of radiology, 2008. 68(3): p. 362-368.

32. Nachtrab, F., et al., Quantitative material analysis by dual-energy computed tomography for industrial NDT applications. Nuclear Instruments and Methods in Physics Research Section A: Accelerators, Spectrometers, Detectors and Associated Equipment, 2011. 633: p. S159-S162.

33. Bonnin, A., et al., Concept of effective atomic number and effective mass density in dual-energy X-ray computed tomography. Nuclear Instruments and Methods in Physics Research Section B: Beam Interactions with Materials and Atoms, 2014. 318: p. 223-231.

34. Helfen, L., et al., High-resolution three-dimensional imaging of flat objects by synchrotronradiation computed laminography. Applied Physics Letters, 2005. 86(7): p. 071915.

35. Helfen, L., et al. Laminographic imaging using synchrotron radiation-challenges and opportunities. in Journal of Physics: Conference Series. 2013. IOP Publishing.

36. Hoshino, M., et al. Development of an X-ray Micro-Laminography System at SPring-8. in AIP Conference Proceedings. 2011. AIP.

37. $\mathrm{Xu}, \mathrm{F}$., et al., Synchrotron radiation computed laminography for polymer composite failure studies. Journal of synchrotron radiation, 2010. 17(2): p. 222-226.

38. O'Brien, N.S., et al., Recent advances in X-ray cone-beam computed laminography. Journal of X-ray science and technology, 2016. 24(5): p. 691-707.

39. Moffat, A.J., et al., In situ synchrotron computed laminography of damage in carbon fibre-epoxy [90/0] s laminates. Scripta Materialia, 2010. 62(2): p. 97-100.

40. Rehak, M., U. Hassler, and R. Hanke. Acquisition trajectories for X-ray tomosynthesis applied to planar samples. in 2nd International Symposium on NDT in Aerospace. 2010.

41. Bull, D., et al., Three-dimensional assessment of low velocity impact damage in particle toughened composite laminates using micro-focus X-ray computed tomography and synchrotron radiation laminography. Composites Part A: Applied Science and Manufacturing, 2013. 52: p. $62-69$.

42. Bullinger, O., et al. Laminographic inspection of large carbon fibre composite aircraft-structures at airbus. in 19th World Conference on Non-Destructive Testing (WCNDT 2016). 2016.

43. Gondrom, S., et al., X-ray computed laminography: an approach of computed tomography for applications with limited access. Nuclear engineering and design, 1999. 190(1-2): p. 141-147.

44. Fu, J., B. Jiang, and B. Li, Large field of view computed laminography with the asymmetric rotational scanning geometry. Science China Technological Sciences, 2010. 53(8): p. 2261-2271.

45. Fisher, S.L., et al., Laminography in the lab: imaging planar objects using a conventional x-ray CT scanner. Measurement Science and Technology, 2019. 30(3): p. 035401.

46. Wood, C., et al., Computed laminography of cfrp using an x-ray cone beam and robotic sample manipulator systems. NDT \& E International, 2017.

47. Helfen, L., et al., Investigation of hybrid pixel detector arrays by synchrotron-radiation imaging. Nuclear Instruments and Methods in Physics Research Section A: Accelerators, Spectrometers, Detectors and Associated Equipment, 2006. 563(1): p. 163-166.

48. Schorr, C., et al., Registration of a priori information for computed laminography. NDT \& E International, 2017. 86: p. 106-112. 
49. Gregor, J. and T. Benson, Computational analysis and improvement of SIRT. IEEE Transactions on Medical Imaging, 2008. 27(7): p. 918-924.

50. Batenburg, K., W. Palenstijn, and J. Sijbers. 3D imaging of semiconductor components by discrete laminography. in AIP Conference Proceedings. 2014. AIP.

51. Helfen, L., et al., On the implementation of computed laminography using synchrotron radiation. Review of Scientific Instruments, 2011. 82(6): p. 063702.

52. Kak, A.C. and M. Slaney, Principles of computerized tomographic imaging. 1988: IEEE press.

53. Biguri, A., et al., TIGRE: a MATLAB-GPU toolbox for CBCT image reconstruction. Biomedical Physics \& Engineering Express, 2016. 2(5): p. 055010.

54. Biguri, A., et al., Arbitrarily large iterative tomographic reconstruction on multiple GPUs using the TIGRE toolbox. arXiv preprint arXiv:1905.03748, 2019.

55. Li, X., D. Zhang, and B. Liu, A generic geometric calibration method for tomographic imaging systems with flat-panel detectors-A detailed implementation guide. Medical physics, 2010. 37(7Part1): p. 3844-3854.

56. Miao, H., et al., A phantom-based calibration method for digital x-ray tomosynthesis. Journal of X-ray science and technology, 2012. 20(1): p. 17-29.

57. Kraemer, A. and G. Lanza, Assessment of the measurement procedure for dimensional metrology with X-ray computed tomography. Procedia CIRP, 2016. 43: p. 362-367.

58. Blumensath, T., N. O'Brien, and C.E. Wood, Calibration of Robotic Manipulator Systems for Cone-Beam Tomography Imaging. IEEE Transactions on Nuclear Science, 2018. 65(7): p. 13841393.

59. Moreno, P. and F. Calderero. Evaluation of Sharpness Measures and Proposal of a Stop Criterion for Reverse Diffusion in the Context of Image Deblurring. in VISAPP (1). 2013. 


\section{Appendix A: Details of sample}

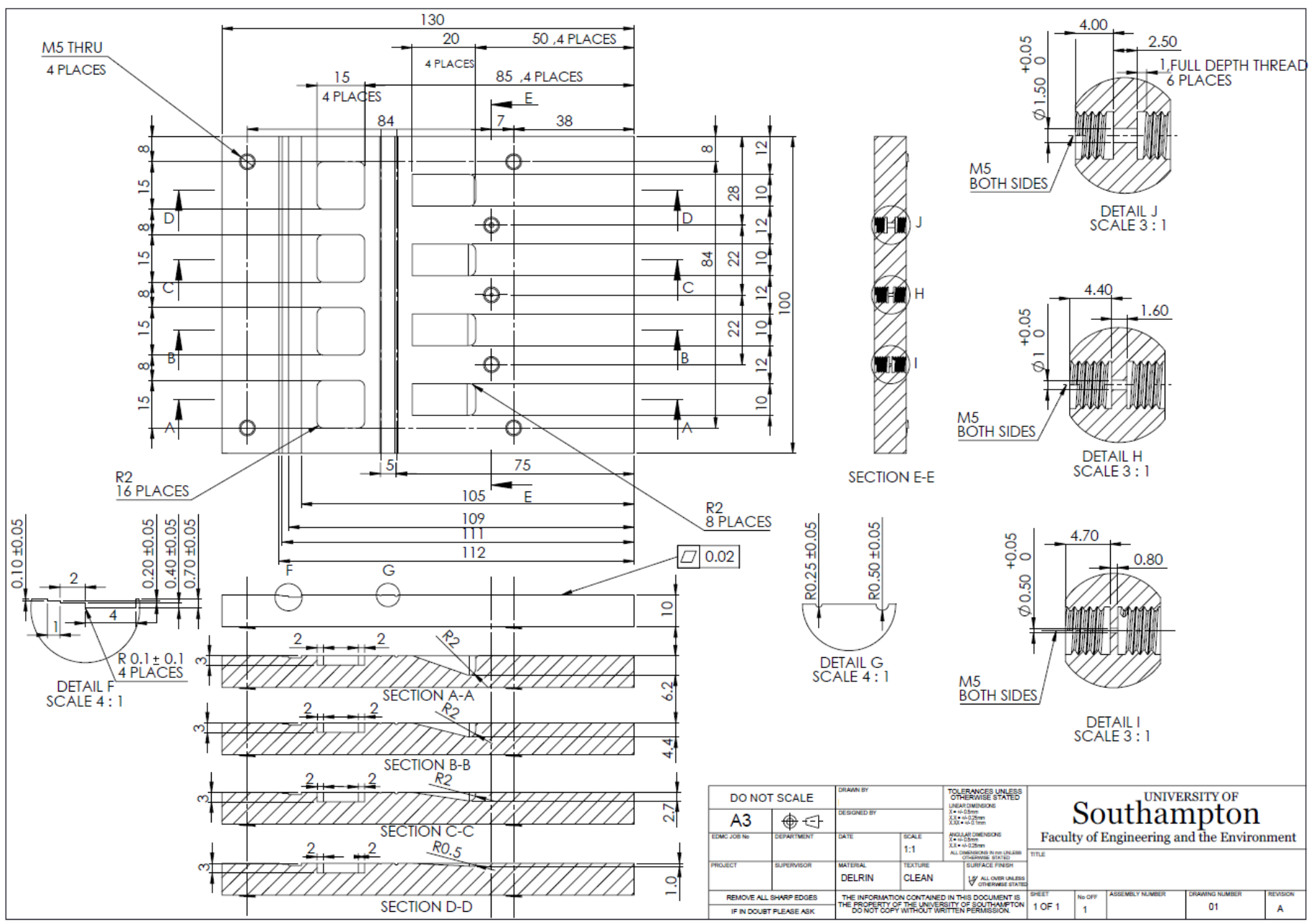

